\title{
Niveles de actividad física de la población colombiana: desigualdades por sexo y condición socioeconómica
}

\author{
Silvia González¹, Olga L. Sarmiento", Óscar Lozano², Andrea Ramírez", Carlos Grijalba ${ }^{1}$ \\ 1 Departamento de Salud Pública, Facultad de Medicina, Universidad de los Andes, Bogotá, D.C., Colombia \\ 2 Departamento Administrativo del Deporte, la Recreación, la Actividad Física y el Aprovechamiento del Tiempo \\ Libre, Coldeportes, Bogotá, D.C., Colombia
}

Introducción. Las desigualdades en los niveles de actividad física son evidentes a nivel mundial. En Colombia, uno de los países más desiguales de Latinoamérica, la información es limitada; por tal razón, es imperativo conocer las disparidades en actividad física en el país para orientar el diseño de programas y políticas públicas encaminadas a promoverla.

Objetivos. Estimar las prevalencias del cumplimiento de recomendaciones sobre actividad física y sus factores asociados, identificar desigualdades por sexo y condición socioeconómica en el cumplimiento de estas recomendaciones en el año 2010 y evaluar la tendencia de las prevalencias de actividad física en un período de cinco años.

Materiales y métodos. Se hizo un análisis secundario de la Encuesta Nacional de la Situación Nutricional, 2005-2010. La muestra total incluyó 27.243 adultos. Los niveles de actividad física se midieron con el cuestionario internacional de actividad física. La condición socioeconómica se midió por el nivel del Sisbén.

Resultados. La prevalencia del cumplimiento de las recomendaciones de actividad física en todos los dominios fue menor entre las mujeres. Los adultos de menor nivel socioeconómico tuvieron la menor prevalencia en "tiempo libre" y la mayor en "uso de la bicicleta como medio de transporte". Los factores asociados con el cumplimiento de las recomendaciones difirieron según el sexo y el dominio de actividad física. Las variables individuales y del hogar explicaron 13,6\% de las desigualdades por sexo y $23,2 \%$ de las desigualdades por nivel socioeconómico. En un período de cinco años la prevalencia de "actividad física en el tiempo libre" disminuyó y aumentó en "caminar como medio de transporte".

Conclusiones. Son preocupantes las bajas prevalencias de cumplimiento de las recomendaciones sobre actividad física en el tiempo libre en las mujeres y las personas de menor nivel socioeconómico. En futuras intervenciones para incrementar los niveles de actividad física deberán considerarse las desigualdades por sexo y condición socioeconómica, así como sus factores asociados.

Palabras clave: desigualdades en la salud, actividad motora, factores socioeconómicos, encuestas nutricionales, adultos, prevalencia.

http://dx.doi.org/10.7705/biomedica.v34i3.2258

Physical activity levels among Colombian adults: Inequalities by gender and socioeconomic status

Introduction: Worldwide studies show inequalities in physical activity levels related to sociodemographic characteristics. In Colombia, among the countries in Latin America with the highest inequality, the evidence related to inequalities in physical activity is limited. It is imperative to identify disparities in physical activity in the country, to guide the design of public policies aimed at promoting physical activity.

Objectives: 1) To estimate the prevalence and associated factors of meeting physical activity recommendations; 2) to assess inequalities by gender and socioeconomic status in meeting physical activity recommendations, and 3 ) to assess the trends in physical activity prevalence within a fiveyear period.

Materials and methods: A secondary analysis of data from the 2010 National Nutrition Survey was conducted. The sample included 27,243 adults. The International Physical Activity Questionnaire was used to measure leisure time and transport domains. Socioeconomic status was measured by the Sisben level.

\section{Contribución de los autores:}

Silvia González y Olga L. Sarmiento: diseño del estudio

Óscar Lozano: análisis de la política pública

Todos los autores participaron en el análisis de resultados y la escritura del artículo. 
Results: Compared to men, women were less likely to meet physical activity recommendations in all domains. Compared to adults from high socioeconomic-status households, low socioeconomic-status adults had a lower prevalence of meeting physical activity recommendations during leisure time and the highest prevalence of using a bicycle for transport. The factors associated with meeting physical activity recommendations differed by gender and physical activity domain. Household and individual variables explained $13.6 \%$ of the inequalities observed by gender, and $23.2 \%$ of the inequalities by socioeconomic status. In a five-year period, the prevalence of physical activity in leisure time decreased, while the physical activity of walking for transport increased and biking for transport did not change.

Conclusions: Future interventions to increase physical activity levels in Colombia must consider inequalities by gender and socioeconomic status. Of special concern is the low prevalence of meeting physical activity recommendations during leisure time in both women and in the low socioeconomic status population.

Key words: Health inequalities, motor activity, socioeconomic factors, nutrition surveys, adults, prevalence. http://dx.doi.org/10.7705/biomedica.v34i3.2258

El rápido aumento en la morbilidad y la mortalidad por enfermedades crónicas no transmisibles constituye uno de los principales problemas de salud pública a nivel global (1). Según la Organización Mundial de la Salud (OMS), para el año 2008, $63,1 \%$ de los 57 millones de muertes ocurridas en el mundo se debieron a las enfermedades crónicas no transmisibles (1). En países de ingresos medios ocurren cerca del $80,0 \%$ de las muertes por este motivo (2). En América Latina, estas enfermedades son las principales causas de muerte y se espera que su prevalencia continúe aumentando. Por ejemplo, se prevé un aumento de $50,0 \%$ de los casos de diabetes para el año 2030 (3).

Frente a esta problemática, la promoción de la actividad física es una inversión vital para la prevención de las enfermedades crónicas no transmisibles (4). La actividad física es un factor protector para las cardiopatías isquémicas, la diabetes mellitus, los trastornos cerebrovasculares y la obesidad (5). En el 2008, cerca de 5,3 millones de los 57 millones de muertes ocurridas en el mundo se atribuyeron a la inactividad física (6). Es preocupante que según las estimaciones, en el 2012 menos del $50,0 \%$ de la población a nivel global cumplía con las recomendaciones de hacer, por lo menos, 30 minutos de actividad física moderada cinco días en la semana, o 20 minutos de actividad vigorosa tres días en la semana, o una combinación igualmente efectiva para alcanzar 600 equivalentes metabólicos (metabolic equivalent

\footnotetext{
Correspondencia:

Olga L. Sarmiento, Departamento de Salud Pública, Facultad de Medicina, Universidad de los Andes, Carrera $3 \mathrm{~N}^{\circ} 18 \mathrm{~A}-10$, piso 8, Edificio Q, Bogotá, D. C., Colombia

Teléfono: (571) 339 4949, extensiones 3798 y 3785

osarmien@uniandes.edu.co

Recibido: 04/02/14; aceptado: 12/05/14
}

of task, METS) por semana (7). En las Américas, $43,0 \%$ de la población es inactiva. Esta prevalencia de inactividad física en las mujeres varía entre $17,0 \%$ en Guatemala y $70,9 \%$ en Argentina, y entre 15,3 y $65,8 \%$ en los hombres en estos mismos países (7). En Colombia, la prevalencia nacional de cumplimiento de las recomendaciones de dedicar 150 minutos a la actividad física moderada por semana fue de $53,5 \%$ en el 2010 (8).

En estudios para evaluar los niveles de actividad física de la población adulta a nivel global, son evidentes las desigualdades por sexo, nivel socioeconómico y nivel educativo (9-12). Las prevalencias de inactividad física en el dominio de tiempo libre son mayores en las mujeres $(33,9$ Vs. $27,9 \%)$ y en la población con menor nivel socioeconómico y educativo (9-12). Estas desigualdades son aún más evidentes en los países en desarrollo (9).

Colombia se considera uno de los países con mayores desigualdades socioeconómicas en América Latina, con un coeficiente de Gini de 0,54 (13). El Plan Nacional de Desarrollo, 2010-2014, ubica la promoción de la actividad física en la línea de "Igualdad de oportunidades para la prosperidad social" (14). Sin embargo, la información actual se limita a estudios locales (15-17) y no se cuenta con información nacional. En este contexto, un análisis de las desigualdades por sexo y condición socioeconómica con representatividad nacional, permitirá establecer estimadores para evaluar los programas y las políticas sobre actividad física.

Uno de los objetivos de desarrollo del milenio es promover la igualdad entre los sexos con el fin de crear sociedades más equitativas (18). En el contexto de la prevención de las enfermedades crónicas no transmisibles mediante la actividad física, es necesario evaluar las desigualdades por sexo y las condiciones socioeconómicas. La 
desigualdad se entiende como la presencia de diferencias en los comportamientos relacionados con la salud, como la actividad física, entre los grupos sociales, lo que predispone a los grupos en desventaja social (mujeres, personas de menor nivel socioeconómico, minorías) a un mayor riesgo en salud (19). Es pertinente evaluar los factores asociados a las desigualdades en la actividad física, con el fin de abordarlos y contribuir a la planeación de intervenciones en salud pública que se reflejen en mayor equidad en la salud y la calidad de vida de la población.

Los objetivos de este estudio fueron estimar las prevalencias de cumplimiento de las recomendaciones sobre actividad física en los dominios de tiempo libre y transporte y sus factores asociados en adultos colombianos durante el 2010; identificar desigualdades por sexo y condición socioeconómica en el cumplimiento de dichas recomendaciones en ese año, y evaluar la tendencia de las prevalencias de actividad física en un período de cinco años. Los resultados servirán como insumo para orientar el diseño de políticas e intervenciones en salud pública encaminadas a promover la actividad física y que, a su vez, aborden la brecha de inequidad.

\section{Materiales y métodos}

\section{Poblaciones de estudio}

Cumplimiento de las recomendaciones sobre actividad física, factores asociados y análisis de desigualdades, 2010. Se hizo un análisis secundario fusionando las bases de datos de la Encuesta Nacional de la Situación Nutricional en Colombia, 2010 (ENSIN) (8) y de la Encuesta Nacional de Demografía y Salud (ENDS), 2010 (20). Es importante resaltar que los hogares incluidos en estas dos encuestas coinciden y que el trabajo de campo se llevó a cabo en el mismo período. La información relacionada con las variables sociodemográficas correspondió a la obtenida por medio del formulario para hogares de la ENDS, 2010 (20). La información sobre actividad física provino de una submuestra de la ENSIN, 2010 (8).

Se aplicó un diseño muestral probabilístico, de conglomerados, estratificado y de varias etapas en la selección de una muestra nacional de 50.670 hogares (20). El componente de actividad física de la ENSIN, 2010, tuvo una muestra de 14.465 adultos de 18 a 64 años residentes en zonas urbanas, representativa a nivel nacional y regional (8).
Análisis de la tendencia de las prevalencias de actividad física, 2005-2010. Para evaluar la tendencia de las prevalencias de actividad física en un período de cinco años, se hizo un análisis secundario de la ENSIN, 2005, y de la ENSIN, 2010. Las muestras correspondientes de cada año incluyeron 12.778 adultos en el 2005 (21) y 14.465 adultos en el 2010 (8).

Las tres encuestas se hicieron en los hogares de los adultos seleccionados, utilizando los sistemas CAPI (Computer Assisted Personal Interviewing) y PDA (Portable Data Assistant). Todos los protocolos fueron aprobados por el Comité de Ética de Profamilia.

\section{Medición de la actividad física}

Las variables dependientes correspondieron al cumplimiento de la recomendación sobre actividad física (sí Vs. no) en dos dominios. Se analizaron los dominios de tiempo libre y transporte, los cuales tienen una mayor validez en poblaciones de América Latina $(22,23)$.

Para medir la actividad física se utilizó la versión larga del cuestionario internacional sobre actividad física (International Physical Activity Questionnaire, IPAQ) desarrollado por la OMS (22-24). Este instrumento se recomienda para la medición de la actividad física en encuestas de población, principalmente en zonas urbanas $(22,23)$. La estructura de los dominios del IPAQ usada en la ENSIN, 2010, fue igual a la utilizada en la ENSIN, 2005, con el fin de garantizar la comparación de los datos. Se usaron las actuales recomendaciones para adultos de la OMS y de los Centers for Disease Control and Prevention (CDC) como punto de corte $(25,26)$.

La construcción de las variables de actividad física siguió las indicaciones del manual del IPAQ (27). El tiempo dedicado a cada actividad reportada se convirtió en minutos por día. Cuando la duración de la actividad física por día superaba los 180 minutos, los valores se truncaron y se les asignó el valor único de 180 minutos. Esta regla permitió un máximo de 21 horas semanales. Para calcular el tiempo total por semana, los minutos de actividad física vigorosa se multiplicaron por dos y se sumaron al número de minutos de actividad física moderada (27). Las variables resultantes fueron las siguientes:1) cumplir con las recomendaciones en cuanto a tiempo libre, que consisten en hacer actividad física moderada en el tiempo libre, al menos, durante 150 minutos por semana, o hacer actividad física vigorosa durante, al menos, 75 
minutos por semana o una combinación equivalente de las dos; 2) cumplir con la recomendación de caminar como medio de transporte, o sea, hacerlo durante al menos 150 minutos por semana, y 3) cumplir con la recomendación de montar en bicicleta como medio de transporte, o sea, hacerlo durante al menos 150 minutos.

\section{Medición de las variables independientes}

Cumplimiento de las recomendaciones sobre actividad física, factores asociados y análisis de desigualdades, 2010. Las variables independientes incluyeron factores sociodemográficos y de salud. Las características sociodemográficas incluyeron sexo (mujer $V s$. hombre), edad (18 a 29 años $V s$. 30 a 49 años $V s .50$ a 64 años), raza (indígena $V s$. afrodescendiente $V s$. otros [mestizos y blancos]), nivel de Sisbén (1 Vs. 2 Vs. 3 Vs. 4 a 6), nivel educativo, considerado como el más alto alcanzado o completado por la persona (ninguno, preescolar o primaria Vs. secundaria Vs. técnico, tecnológico, universidad o posgrado) y tipo de familia (unipersonal o nuclear incompleta Vs. extensa). Como indicador de la condición laboral se incluyó la variable ocupación en la semana anterior (trabajó o tiene trabajo Vs. buscó trabajo Vs. estudió Vs. oficios del hogar $V s$. pensionado u otro). El índice del Sisbén es una medida del estándar de vida en Colombia que permite establecer un patrón frente al cual se valoran las condiciones de vida de los hogares (28). Se usó la versión del Sisbén III y se tomaron como referencia los puntos de corte del Ministerio de la Protección Social, los cuales clasifican los hogares en cuatro niveles (1, 2, 3 y 4 o más).

Los factores de salud incluyeron el índice de masa corporal (IMC) (delgadez Vs. normal Vs. sobrepeso Vs. obesidad); para estimar el IMC se pesó con balanzas electrónicas marca Seca, referencia 872 , con una capacidad de $200 \mathrm{~kg}$ y una precisión de $50 \mathrm{~g}$ para los pesos de 0 a $50 \mathrm{~kg}$ y de $100 \mathrm{~g}$ para los pesos de $50 \mathrm{~kg}$ a $200 \mathrm{~kg}$. La talla se midió con tallímetros portátiles (Diseños Flores y Weight and Measure) de una longitud máxima de $2 \mathrm{~m}$ y una precisión de $1 \mathrm{~mm}$. La condición de discapacidad se registró según el propio reporte de las personas (sí Vs. no) e incluyó cualquier tipo de discapacidad física o sensorial.

\section{Análisis estadístico}

Cumplimiento de las recomendaciones sobre actividad física, factores asociados y análisis de las desigualdades, 2010. El análisis estadístico incluyó cuatro fases. En la primera se calcularon las prevalencias de actividad física en los dominios de tiempo libre y caminar o montar bicicleta como medio de transporte. En la segunda fase, se estratificó por las variables sociodemográficas y de salud. Las diferencias entre las prevalencias de las categorías de las variables independientes se evaluaron con la prueba de ji al cuadrado de Pearson. En la tercera fase, con el fin de estimar razones de prevalencia, se utilizó un modelo de regresión de Poisson con varianza robusta (29). En la cuarta fase, se calculó el cambio porcentual en los estimadores de sexo y nivel socioeconómico del modelo bivariado al multivariado, para identificar la contribución de las variables individuales y del hogar en las desigualdades observadas (30), de acuerdo con la siguiente fórmula:

$$
\frac{\text { RP crudo }- \text { RP ajustado }}{R P \text { crudo }} * 100
$$

donde RP crudo es la razón de prevalencia estimada para el nivel del Sisbén más bajo o para las mujeres en el modelo bivariado y RP ajustado es la razón de prevalencia estimada para estas mismas categorías en el modelo multivariado.

\section{Análisis de la tendencia de las prevalencias de actividad física, 2005-2010}

Se compararon los estimadores de prevalencia en los dominios de actividad física obtenidos en la ENSIN, 2005, y la ENSIN, 2010, mediante un análisis de homogeneidad con la prueba $Q$ de Cochran (31). Todos los análisis bivariados y multivariados se hicieron estratificando por sexo, excepto el análisis para evaluar las desigualdades por sexo.

Todos los análisis estadísticos se ajustaron según los pesos muestrales y el diseño de la muestra, y se hicieron utilizando los programas estadísticos SAS 9.2 y Stata 11.

\section{Resultados}

\section{Cumplimiento de las recomendaciones sobre actividad física, factores asociados y análisis de desigualdades, 2010}

Características sociodemográficas. La edad promedio de la población de adultos estudiada fue de 37,7 años ( $D E=12,9)$. El $57,7 \%$ de los adultos incluidos eran mujeres. Aproximadamente, el $12,0 \%$ de la población pertenecía a minorías raciales. Más del $50,0 \%$ de la población se clasificó en los niveles 1, 2 y 3 del Sisbén, lo que concuerda con el nivel socioeconómico general de 
la población colombiana. Cerca de una cuarta parte de la población tenía un nivel educativo inferior a la secundaria. Solo el $61,1 \%$ de la población adulta reportó tener trabajo, $24,2 \%$ se dedicaba a los oficios del hogar y el $6,7 \%$ se encontraba estudiando. La mayoría de los adultos pertenecía a familias extensas. De los adultos entrevistados, $6,1 \%$ reportó algún tipo de discapacidad física o sensorial. Reflejando la doble carga en malnutrición, $3,1 \%$ de los adultos tenía bajo peso, mientras que $35,5 \%$ tenía sobrepeso y, 17,2 \%, obesidad (cuadro 1).

\section{Prevalencias de cumplimiento de las recomendaciones sobre actividad física}

La prevalencia global de cumplimiento de la recomendación sobre actividad física fue de 53,5\% $\left(\mathrm{IC}_{95 \%} 52,4-54,5\right)$. En el dominio de tiempo libre, la prevalencia fue de 19,9\% (IC $\left.{ }_{95 \%} 19,1-20,8\right)$. En el dominio de transporte, la prevalencia fue de $33,8 \%$ $\left(\mathrm{IC}_{95 \%} 32,8-34,9\right)$ para caminar y de $5,6 \%\left(\mathrm{IC}_{95 \%}\right.$ $5,1-6,1)$ para montar en bicicleta.

\section{Desigualdades en las prevalencias de cumplimiento de las recomendaciones sobre actividad física}

Como reflejo de la desigualdad por sexo, las mujeres tuvieron menores prevalencias de actividad física en todos los dominios, en comparación con los hombres. Esta disparidad fue más significativa en el tiempo libre (mujeres: $13,8 \%$ Vs. hombres: $28,2 \%, p<0,0001)$ y en usar la bicicleta como medio de transporte (mujeres: 1,6\% Vs. hombres: $11,0 \%, p<0,0001)$, en comparación con caminar como medio de transporte (mujeres: $31,2 \% \mathrm{Vs}$. hombres: $37,4 \%, p<0,0001$ ) (cuadros 2 y 3 ). Esta desigualdad también se observó en el uso de la bicicleta durante un mínimo de 10 minutos (mujeres: $5,1 \%$ Vs. hombres: $21,6 \%, p<0,0001$ ) (figura 1).

Además, como reflejo de las desigualdades por estrato socioeconómico, se observaron diferencias significativas según los dominios de actividad física. En el dominio de tiempo libre, en los niveles del Sisbén 1, 2 y 3 se presentó una menor prevalencia de cumplimiento de las recomendaciones, en comparación con los niveles 4 a $6(16,1 \% \mathrm{Vs}$. $19,7 \%$ Vs. $19,8 \%$ Vs. $22,5 \%, p<0,0001)$ de la población. En contraste, en el dominio de montar en bicicleta como medio de transporte, se observó una menor prevalencia en los niveles 4 a 6 del Sisbén, en comparación con el nivel 1 (4,5\% Vs. $7,4 \%$ ) (figura 2).
Cuadro 1. Características demográficas de 14.465 personas de 18 a 64 años residentes en zonas urbanas de Colombia. Encuesta Nacional de la Situación Nutricional en Colombia (ENSIN), 2010, y Encuesta Nacional de Demografía y Salud (ENDS), 2010

\begin{tabular}{|c|c|c|c|}
\hline Características & Número & $\%$ & $\mathrm{EE}^{\mathrm{a}}$ \\
\hline \multicolumn{4}{|l|}{ Edad (años) } \\
\hline $18-29$ & 4.828 & 33,4 & 0,50 \\
\hline $30-49$ & 6.531 & 44,8 & 0,55 \\
\hline $50-64$ & 3.106 & 21,8 & 0,45 \\
\hline \multicolumn{4}{|l|}{ Sexo } \\
\hline Mujeres & 8.224 & 57,7 & 0,49 \\
\hline Hombres & 6.241 & 42,3 & 0,49 \\
\hline \multicolumn{4}{|l|}{$\operatorname{Raza}^{\mathrm{b}}$} \\
\hline Indígena & 811 & 2,6 & 0,17 \\
\hline Afrodescendiente ${ }^{c}$ & 1.441 & 9,8 & 0,39 \\
\hline Otros $^{d}$ & 12.112 & 87,7 & 0,42 \\
\hline \multicolumn{4}{|l|}{ Nivel de Sisbén } \\
\hline Nivel 1 & 4.935 & 29,3 & 0,66 \\
\hline Nivel 2 & 1.974 & 13,3 & 0,42 \\
\hline Nivel 3 & 1.896 & 11,4 & 0,38 \\
\hline Niveles 4 a 6 & 5.660 & 46,0 & 0,75 \\
\hline \multicolumn{4}{|l|}{ Nivel de escolaridade } \\
\hline Ninguna, preescolar o primaria & 4.341 & 26,8 & 0,52 \\
\hline Secundaria & 6.691 & 47,3 & 0,57 \\
\hline $\begin{array}{l}\text { Técnico, tecnológico, universidad } \\
\text { o posgrado }\end{array}$ & 3.385 & 26,0 & 0,62 \\
\hline \multicolumn{4}{|l|}{ Ocupación en la semana anterior ${ }^{\mathrm{e}}$} \\
\hline Trabajó o tiene trabajo & 8.858 & 61,1 & 0,51 \\
\hline Buscó trabajo & 542 & 3,9 & 0,21 \\
\hline Estudió & 862 & 6,7 & 0,28 \\
\hline Oficios del hogar & 3.614 & 24,2 & 0,45 \\
\hline Pensionado u otro & 585 & 4,2 & 0,21 \\
\hline \multicolumn{4}{|l|}{ Tipo de familia } \\
\hline Unipersonal o nuclear incompleta ${ }^{\dagger}$ & 1.833 & 12,5 & 0,39 \\
\hline Extensag & 12.632 & 87,5 & 0,39 \\
\hline \multicolumn{4}{|l|}{ Condición de discapacidad ${ }^{h}$} \\
\hline Sí & 852 & 6,1 & 0,29 \\
\hline No & 13.613 & 93,9 & 0,29 \\
\hline \multicolumn{4}{|l|}{ Índice de masa corporal } \\
\hline Delgadez & 407 & 3,1 & 0,19 \\
\hline Normal & 5.779 & 44,3 & 0,54 \\
\hline Sobrepeso & 4.850 & 35,5 & 0,52 \\
\hline Obesidad & 2.504 & 17,2 & 0,40 \\
\hline
\end{tabular}

a EE: error estándar

b Se excluyeron los gitanos o Rom al no tener una muestra suficiente para obtener resultados estadísticamente significativos. El número de observaciones se vio afectado por los valores ausentes.

c Comprende los siguientes grupos: negro, mulato, afrocolombiano, afrodescendiente, raizal del archipiélago de San Andrés o palenquero de San Basilio.

${ }^{d}$ Comprende mestizos y blancos.

- Se agruparon algunas categorías considerando que no existían diferencias significativas entre las variables agrupadas.

${ }^{\dagger}$ Hogares conformados por una sola persona o familias con uno solo de los padres

${ }^{9}$ Familias nucleares con los dos padres, y familias con padres, hijos y otros parientes

${ }^{\mathrm{h}}$ Incluye cualquier tipo de discapacidad física o sensorial.

Con respecto al uso de la bicicleta por un período mínimo de 10 minutos consecutivos, se observó una prevalencia general de $12,1 \%$; se encontró que $21,6 \%$ de los hombres y $5,1 \%$ de las mujeres montan en bicicleta por lo menos 10 minutos consecutivos. 
Cuadro 2. Modelo multivariado para el cumplimiento de las recomendaciones sobre actividad física en tiempo libre, caminar como medio de transporte y usar la bicicleta como medio de transporte en mujeres. Encuesta Nacional de la Situación Nutricional en Colombia (ENSIN), 2010, y Encuesta Nacional de Demografía y Salud (ENDS), 2010

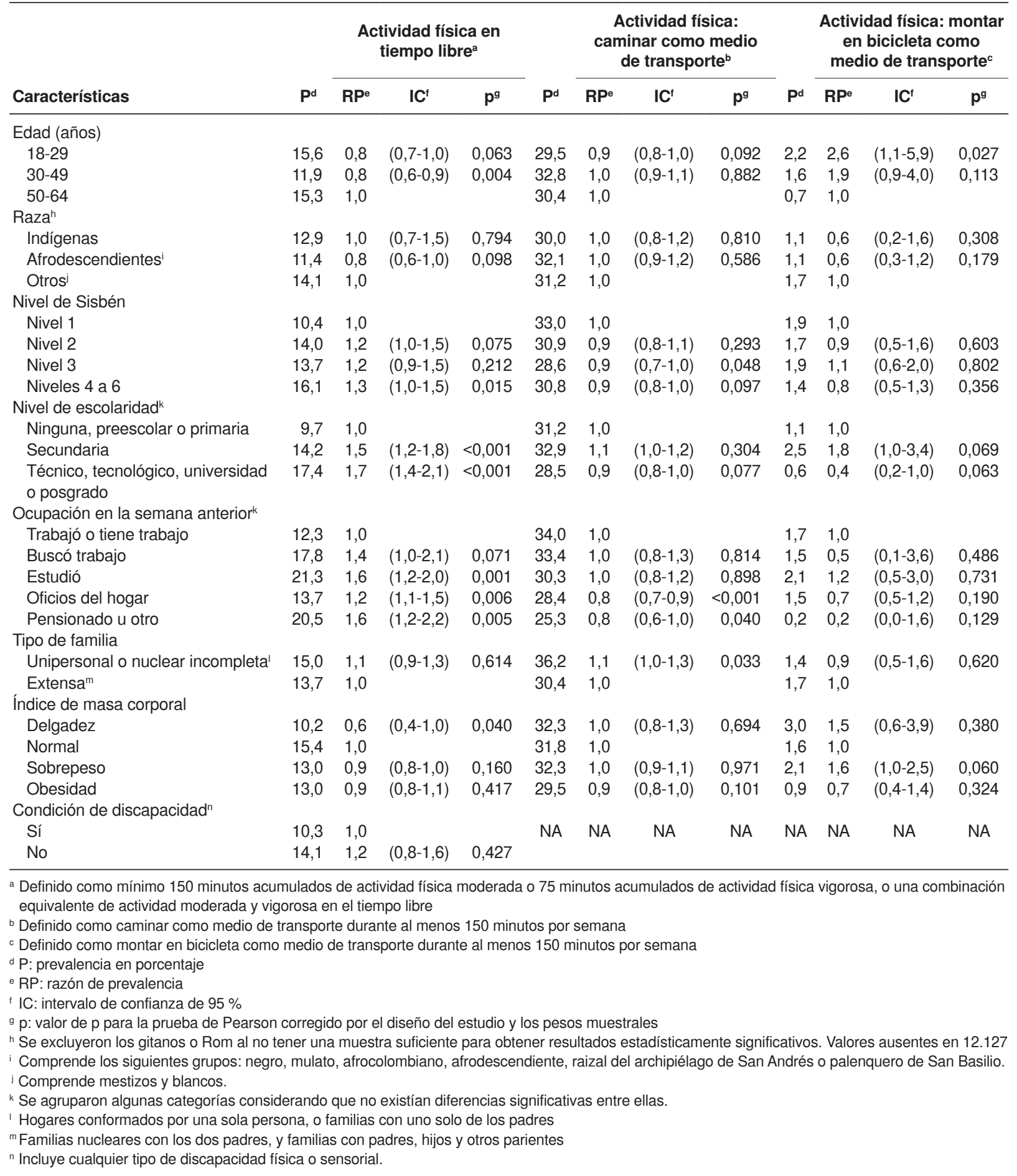


Cuadro 3. Modelo multivariado para el cumplimiento de las recomendaciones sobre actividad física en el tiempo libre, caminar como medio de transporte y usar la bicicleta como medio de transporte en hombres. Encuesta Nacional de la Situación Nutricional en Colombia (ENSIN), 2010, y Encuesta Nacional de Demografía y Salud (ENDS), 2010

\begin{tabular}{|c|c|c|c|c|c|c|c|c|c|c|c|c|}
\hline \multirow[b]{2}{*}{ Características } & \multirow[b]{2}{*}{$P^{d}$} & \multicolumn{3}{|c|}{$\begin{array}{l}\text { Actividad física } \\
\text { en tiempo libre }\end{array}$} & \multirow[b]{2}{*}{$\mathbf{P}^{\mathbf{d}}$} & \multicolumn{3}{|c|}{$\begin{array}{l}\text { Actividad física: } \\
\text { caminar como medio } \\
\text { de transporte }^{\mathrm{b}}\end{array}$} & \multirow[b]{2}{*}{$\mathbf{P}^{\mathbf{d}}$} & \multicolumn{3}{|c|}{$\begin{array}{l}\text { Actividad física: } \\
\text { usar la bicicleta como } \\
\text { medio de transporte }^{c}\end{array}$} \\
\hline & & $\mathrm{RP}^{\mathrm{e}}$ & $I^{f}$ & $\mathbf{p}^{g}$ & & $\mathbf{R P}^{\mathrm{e}}$ & $I^{f}$ & $p^{g}$ & & $\mathbf{R P}$ & $I^{f}$ & $\mathbf{p}^{g}$ \\
\hline \multicolumn{13}{|l|}{ Edad (años) } \\
\hline $18-29$ & 38,9 & 1,7 & $(1,4-2,0)$ & $<0,001$ & 34,5 & 0,8 & $(0,7-0,9)$ & 0,002 & 10,9 & 0,9 & $(0,7-1,2)$ & 0,631 \\
\hline $30-49$ & 23,4 & 1,2 & $(1,0-1,4)$ & 0,031 & 37,5 & 0,9 & $(0,8-1,0)$ & 0,247 & 10,9 & 0,9 & $(0,7-1,2)$ & 0,598 \\
\hline $50-64$ & 20,3 & 1,0 & & & 41,8 & 1,0 & & & 11,2 & 1,0 & & \\
\hline \multicolumn{13}{|l|}{ Raza $^{h}$} \\
\hline Indígenas & 30,6 & 1,2 & $(0,9-1,6)$ & 0,148 & 40,8 & 1,1 & $(0,9-1,3)$ & 0,415 & 15,4 & 1,3 & $(0,9-2,0)$ & 0,223 \\
\hline Afrodescendientes ${ }^{i}$ & 28,6 & 0,9 & $(0,8-1,1)$ & 0,489 & 33,4 & 0,9 & $(0,8-1,0)$ & 0,101 & 10,5 & 0,9 & $(0,7-1,2)$ & 0,380 \\
\hline Otros $^{j}$ & 28,2 & 1,0 & & & 37,7 & 1,0 & & & 10,9 & 1,0 & & \\
\hline \multicolumn{13}{|l|}{ Nivel de Sisbén } \\
\hline Nivel 1 & 24,2 & 1,0 & & & 35,2 & 1,0 & & & 15,2 & 1,0 & & \\
\hline Nivel 2 & 28,0 & 1,1 & $(1,0-1,4)$ & 0,136 & 40,0 & 1,1 & $(1,0-1,3)$ & 0,126 & 10,1 & 0,8 & $(0,6-1,0)$ & 0,080 \\
\hline Nivel 3 & 28,3 & 1,1 & $(0,9-1,3)$ & 0,279 & 36,4 & 1,0 & $(0,9-1,2)$ & 0,854 & 10,9 & 0,9 & $(0,7-1,2)$ & 0,402 \\
\hline Niveles 4 a 6 & 30,7 & 1,1 & $(1,0-1,3)$ & 0,052 & 38,2 & 1,1 & $(0,9-1,2)$ & 0,372 & 8,7 & 0,7 & $(0,6-0,9)$ & 0,005 \\
\hline \multicolumn{13}{|l|}{ Nivel de escolaridadk } \\
\hline Ninguna, preescolar o primaria & 19,6 & 1,0 & & & 37,7 & 1,0 & & & 14,4 & 1,0 & & \\
\hline Secundaria & 30,1 & 1,3 & $(1,1-1,5)$ & 0,001 & 36,6 & 1,0 & $(0,9-1,1)$ & 0,980 & 11,8 & 0,9 & $(0,7-1,1)$ & 0,294 \\
\hline $\begin{array}{l}\text { Técnico, tecnológico, universidad } \\
\text { o posgrado }\end{array}$ & 33,8 & 1,3 & $(1,1-1,6)$ & 0,001 & 38,2 & 1,0 & $(0,9-1,2)$ & 0,724 & 5,9 & 0,5 & $(0,3-0,6)$ & $<0,001$ \\
\hline \multicolumn{13}{|l|}{ Ocupación en la semana anteriork } \\
\hline Trabajó o tiene trabajo & 24,2 & 1,0 & & & 37,4 & 1,0 & & & 11,7 & 1,0 & & \\
\hline Buscó trabajo & 41,1 & 1,6 & $(1,4-1,9)$ & $<0,001$ & 42,8 & 1,1 & $(1,0-1,4)$ & 0,115 & 9,8 & 0,8 & $(0,5-1,1)$ & 0,165 \\
\hline Estudió & 51,1 & 1,6 & $(1,3-1,8)$ & $<0,001$ & 35,9 & 1,0 & $(0,8-1,2)$ & 0,766 & 8,3 & 0,8 & $(0,5-1,3)$ & 0,413 \\
\hline Oficios del hogar & 40,9 & 1,7 & $(1,4-2,2)$ & $<0,001$ & 32,6 & 0,9 & $(0,7-1,2)$ & 0,389 & 11,3 & 0,9 & $(0,5-1,6)$ & 0,748 \\
\hline Pensionado u otro & 36,5 & 1,8 & $(1,5-2,1)$ & $<0,001$ & 34,6 & 0,9 & $(0,8-1,1)$ & 0,528 & 4,7 & 0,4 & $(0,2-0,7)$ & 0,001 \\
\hline \multicolumn{13}{|l|}{ Tipo de familia } \\
\hline Unipersonal o nuclear incompleta' & 30,4 & 1,0 & $(0,9-1,2)$ & 0,823 & 36,7 & 1,0 & $(0,8-1,1)$ & 0,708 & 11,4 & 0,9 & $(0,7-1,3)$ & 0,661 \\
\hline Extensam $^{\mathrm{m}}$ & 28,0 & 1,0 & & & 37,4 & 1,0 & & & 10,9 & 1,0 & & \\
\hline \multicolumn{13}{|l|}{ Índice de masa corporal } \\
\hline Delgadez & 37,7 & 1,0 & $(0,8-1,3)$ & 0,902 & 40,8 & 1,1 & $(0,9-1,4)$ & 0,382 & 16,0 & 1,3 & $(0,8-1,9)$ & 0,314 \\
\hline Normal & 30,6 & 1,0 & & & 38,6 & 1,0 & & & 13,6 & 1,0 & & \\
\hline Sobrepeso & 26,9 & 1,0 & $(0,9-1,1)$ & 0,659 & 37,9 & 0,9 & $(0,9-1,0)$ & 0,205 & 8,6 & 0,7 & $(0,5-0,8)$ & 0,000 \\
\hline Obesidad & 24,1 & 0,9 & $(0,8-1,1)$ & 0,359 & 34,7 & 0,9 & $(0,8-1,0)$ & 0,052 & 7,1 & 0,5 & $(0,4-0,8)$ & 0,001 \\
\hline \multicolumn{13}{|l|}{ Condición de discapacidad" } \\
\hline Sí & 21,7 & 1,0 & & & NA & NA & NA & NA & NA & NA & NA & NA \\
\hline No & 28,7 & 1,2 & $(1,0-1,6)$ & 0,114 & & & & & & & & \\
\hline
\end{tabular}

a Definido como mínimo 150 minutos acumulados de actividad física moderada o 75 minutos acumulados de actividad física vigorosa, o una combinación equivalente de actividad moderada y vigorosa en el tiempo libre

b Definido como caminar como medio de transporte durante al menos 150 minutos por semana

c Definido como usar la bicicleta como medio de transporte durante al menos 150 minutos por semana

d P: prevalencia en porcentaje

e RP: razón de prevalencia

f IC: intervalo de confianza de $95 \%$

${ }^{g}$ p: valor de p para la prueba de Pearson corregido por el diseño del estudio y los pesos muestrales

h Se excluyeron gitanos o Rom al no tener una muestra suficiente para obtener resultados estadísticamente significativos. Valores ausentes en 12.127

' Comprende los siguientes grupos: negro, mulato, afrocolombiano, afrodescendiente, raizal del archipiélago de San Andrés o palenquero de San Basilio.

j Comprende mestizos y blancos.

k Se agruparon algunas categorías considerando que no existían diferencias significativas entre ellas.

' Hogares conformados por una sola persona, o familias con uno solo de los padres

${ }^{m}$ Familias nucleares con los dos padres, y familias con padres, hijos y otros parientes

${ }^{\mathrm{n}}$ Incluye cualquier tipo de discapacidad física o sensorial. 


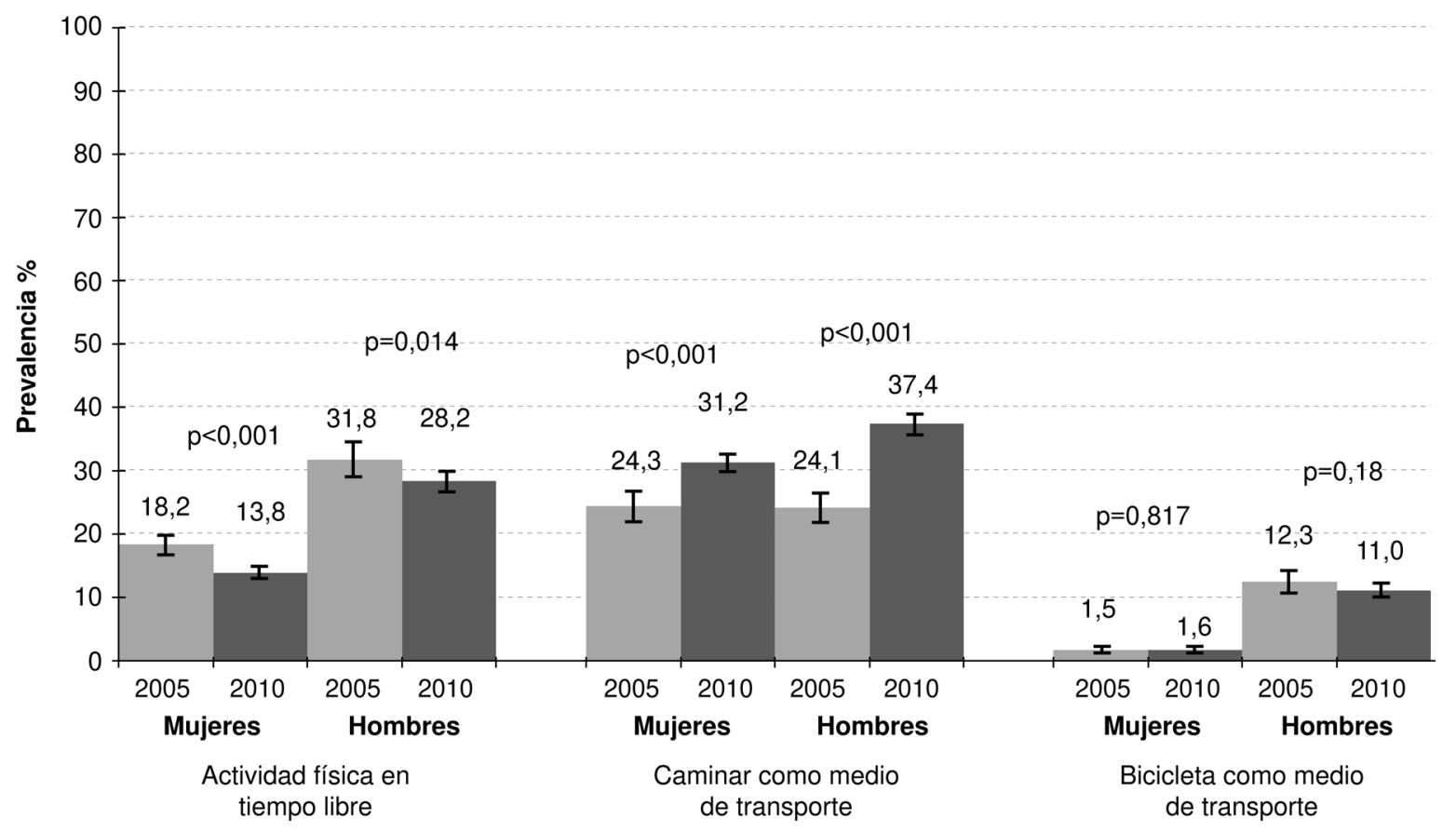

Figura 1. Comparación de las prevalencias de cumplimiento de las recomendaciones sobre actividad física en el 2005 y el 2010 en hombres y mujeres en los dominios de tiempo libre, caminar como medio de transporte y usar la bicicleta como medio de transporte

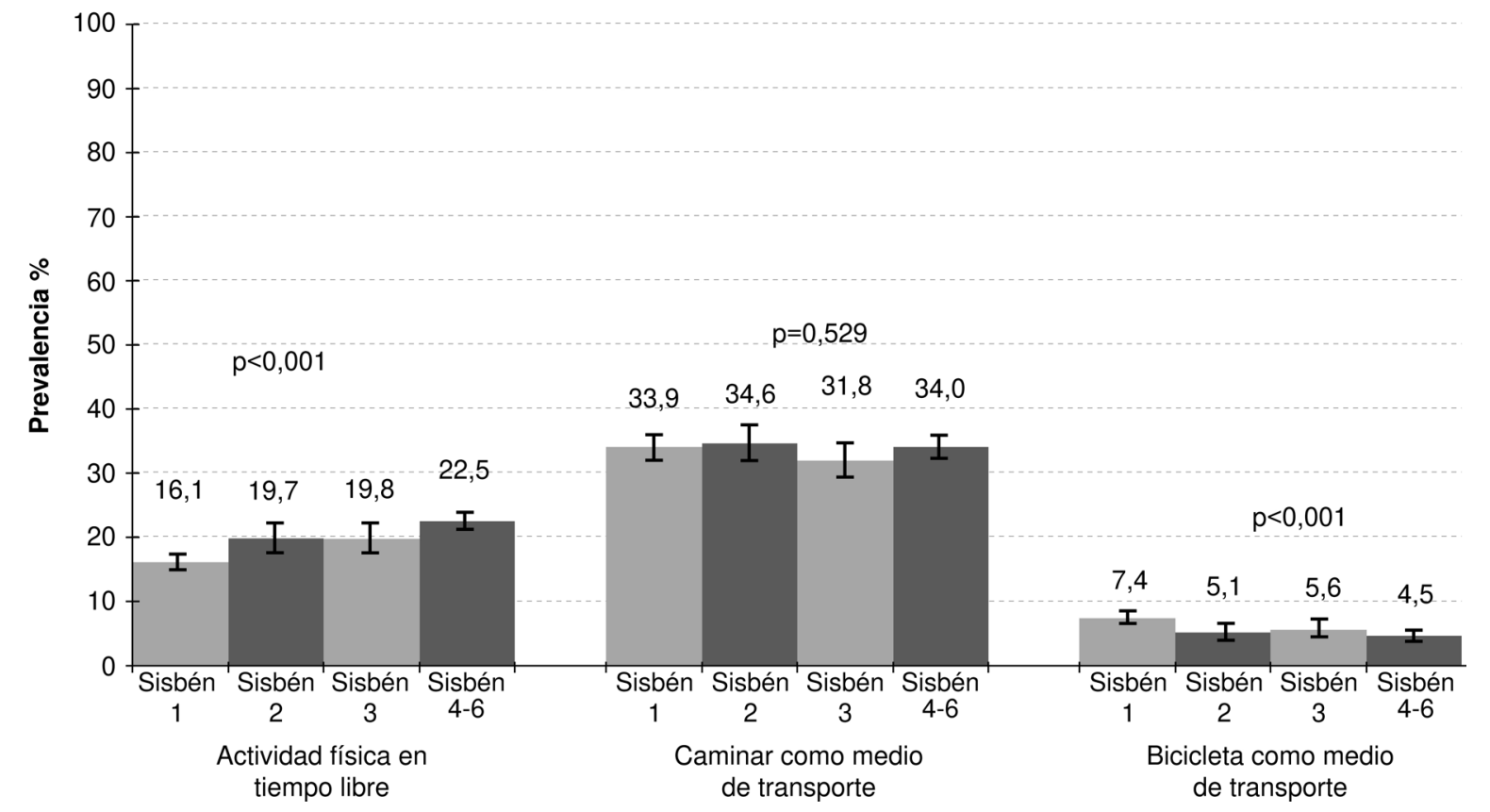

Figura 2. Prevalencias de cumplimiento de las recomendaciones sobre actividad física según nivel socioeconómico del Sisbén en los dominios de tiempo libre, caminar como medio de transporte y usar la bicicleta como medio de transporte 


\section{Factores asociados con el cumplimiento de las recomendaciones sobre actividad física en las mujeres}

Dominio de tiempo libre. En el análisis bivariado se observó que los factores asociados positivamente con el cumplimiento de las recomendaciones para el tiempo libre fueron pertenecer a los niveles $4 \mathrm{a}$ 6 del Sisbén, tener un alto nivel educativo y estar estudiando o ser pensionado, en comparación con los grupos de referencia respectivos. Por el contrario, los factores asociados negativamente con dicho cumplimiento fueron la edad (30-49 años) y tener sobrepeso, en comparación con los respectivos grupos de referencia.

En el análisis multivariado se observó el mismo patrón de asociaciones positivas y negativas. Además, dedicarse a los oficios del hogar tuvo una asociación positiva en comparación con estar trabajando, mientras que un IMC indicativo de delgadez tuvo una asociación negativa con el cumplimiento de las recomendaciones en este dominio, comparado con el peso normal (cuadro 2).

Dominio de transporte: caminar como medio de transporte. En el análisis bivariado el factor asociado positivamente con el cumplimiento de las recomendaciones sobre caminar como medio de transporte por parte de las mujeres fue pertenecer a una familia unipersonal o nuclear incompleta, en comparación con pertenecer a una familia extensa. En contraste, estar pensionada o dedicada a oficios del hogar tuvo una asociación negativa con el cumplimiento de esta recomendación, en comparación con estar trabajando.

En el análisis multivariado se mantuvieron las mismas asociaciones. Además, pertenecer al nivel 3 del Sisbén tuvo una asociación negativa comparado con el nivel de Sisbén 1 (cuadro 2).

Usar la bicicleta como medio de transporte. Con respecto a montar bicicleta como medio de transporte, en el análisis bivariado se observó que los factores asociados positivamente con cumplir las recomendaciones en este dominio fueron la edad (18-29 años y 30-49 años) y tener un nivel educativo máximo de secundaria, en comparación con los respectivos grupos de referencia. Por el contrario, el factor asociado negativamente fue estar pensionada, en comparación con estar trabajando.

En el modelo multivariado se mantiene únicamente la asociación positiva con tener menor edad (18-29 años) comparada con la edad de referencia (50-64 años) (cuadro 2).

\section{Factores asociados el cumplimiento de las recomendaciones sobre actividad física en los hombres}

Dominio de tiempo libre. En el análisis bivariado se observó que los factores que aumentaban la probabilidad de cumplir las recomendaciones para el tiempo libre fueron la edad (18-29 años), pertenecer a los niveles 4 a 6 del Sisbén, tener un nivel educativo de secundaria o superior, estar estudiando, buscando trabajo, dedicado a oficios del hogar o pensionado y no tener discapacidad, en comparación con los grupos de referencia respectivos. Por el contrario, los factores que se asociaron negativamente fueron el sobrepeso y la obesidad, comparados con tener un índice de masa corporal normal.

En el modelo multivariado se observó el mismo patrón de asociaciones positivas, excepto por las asociaciones con el nivel del Sisbén y la discapacidad, las cuales no fueron estadísticamente significativas (cuadro 3).

Dominio de transporte: caminar como medio de transporte. En el modelo bivariado, el único factor asociado negativamente con cumplir las recomendaciones sobre caminar como medio de transporte fueron las edades de 18 a 29 y de 30 a 49 años, en comparación con la asociación negativa con la edad de 50 a 64 años. En el modelo multivariado se observó esta asociación negativa con el grupo de edad de 18 a 29 años, en comparación con el de referencia (50-64 años) (cuadro 3).

Usar la bicicleta como medio de transporte. Con respecto a usar la bicicleta como medio de transporte, en el modelo bivariado se observó que los factores asociados negativamente con la probabilidad de cumplir con las recomendaciones fueron pertenecer a los niveles 4 a 6 del Sisbén, tener un nivel educativo de secundaria o superior, estar pensionado y tener sobrepeso u obesidad, en comparación con sus respectivos grupos de referencia. En el modelo multivariado se observó el mismo patrón de asociaciones negativas (cuadro 3).

\section{Contribución de las variables individuales y del hogar a las desigualdades en el cumplimiento de las recomendaciones sobre actividad física por sexo}

En el dominio de actividad física en el tiempo libre y de caminar como medio de transporte, el 6,4 y el $6,7 \%$ de las desigualdades entre hombres y mujeres se explicaron por las variables individuales y del hogar, respectivamente. En el dominio de 
montar bicicleta como medio de transporte, 13,6\% de las desigualdades entre hombres y mujeres respondió a estas variables.

Contribución de las variables individuales y del hogar a las desigualdades en el cumplimiento de las recomendaciones sobre actividad física por nivel socioeconómico

Esta contribución fue diferente para hombres y mujeres. Para las mujeres, en el dominio de actividad física en el tiempo libre, $23,2 \%$ de la desigualdad entre el nivel del Sisbén más bajo y el más alto, se explicó por variables individuales y del hogar. En el dominio de caminar como medio de transporte, únicamente $2,1 \%$ de la desigualdad respondió a estas variables, y en el dominio de montar en bicicleta como medio de transporte, $6,9 \%$ de la desigualdad por nivel socioeconómico se explicó por variables individuales y del hogar.

En el dominio de la actividad física en tiempo libre entre los hombres, $10,9 \%$ de la desigualdad entre el nivel del Sisbén más bajo y el más alto se explicó por variables individuales y del hogar. En el dominio de caminar como medio de transporte, únicamente $2,9 \%$ de la desigualdad respondió a estas variables, y en el dominio de montar en bicicleta como medio de transporte, $21,56 \%$ de la desigualdad por nivel socioeconómico se explicó por variables individuales y del hogar.

Tendencias de los patrones de actividad física
en un período de cinco años, ENSIN 2005-2010

Las prevalencias de actividad física en el año 2005 en las mujeres, comparadas con los hombres, fueron significativamente menores en actividad física en el tiempo libre y en el dominio de montar en bicicleta como medio de transporte. Preocupa constatar que en el 2010 se mantuvieron las diferencias por sexo con menores prevalencias (figura 1). Al observar los cambios porcentuales en el período de cinco años, se evidenció una disminución en las prevalencias de actividad física en el tiempo libre. Como reflejo de la desigualdad por sexo, el cambio porcentual en esta disminución fue mayor en las mujeres en comparación con los hombres $(4,4 \mathrm{Vs}$. 3,6$)$. Tanto en hombres como en mujeres se evidenció un aumento significativo entre el 2005 y el 2010 en el dominio de caminar como medio de transporte. No se observaron cambios significativos en el uso de la bicicleta como medio de transporte (figura 1).

\section{Discusión}

Como reflejo de las inequidades de Colombia, nuestros resultados evidencian diferencias por sexo y nivel socioeconómico según el dominio de actividad física. Es preocupante observar que las mujeres y quienes pertenecen al nivel del Sisbén más bajo tuvieron las prevalencias más bajas en el cumplimiento de las recomendaciones en el dominio de tiempo libre. En el dominio de transporte, el uso de la bicicleta presentó un patrón diferente que indica que las mujeres y quienes pertenecen al mayor nivel del Sisbén presentaron las menores prevalencias. En cuanto a las desigualdades por nivel socioeconómico, se observó que entre 2,1 y $23,2 \%$ de ellas respondió a variables individuales y del hogar. La proporción de las desigualdades por sexo explicadas por estas variables sugiere que puede haber otras variables no incluidas en este análisis que las expliquen en mayor medida.

De acuerdo con el comportamiento de los niveles de actividad física en el período de 2005 a 2010, es preocupante observar que la prevalencia de actividad física en el tiempo libre ha disminuido significativamente y las desigualdades por sexo se han mantenido constantes en todos los dominios. Sin embargo, es interesante observar la tendencia hacia el aumento en el dominio de caminar como medio de transporte. La prevalencia del uso de la bicicleta como medio de transporte no tuvo cambios significativos en este período.

Las desigualdades observadas entre hombres y mujeres en todos los dominios de actividad física son congruentes con los patrones observados por Hallal, et al., en un análisis a nivel global (7). En el contexto de América Latina, nuestros resultados coinciden con los observados en Brasil con respecto a una menor prevalencia de actividad física en mujeres que en hombres (11), y a una menor prevalencia del cumplimiento de las recomendaciones sobre actividad física en el tiempo libre en los niveles socioeconómicos más bajos (32).

Con respecto a las tendencias de la prevalencia de actividad física a lo largo del tiempo, nuestros resultados sugieren que los patrones observados en Colombia difieren del comportamiento en otros países. Mientras que en Colombia ha disminuido la prevalencia de actividad física en el tiempo libre, a nivel mundial se han evidenciado incrementos en este dominio, principalmente en países de ingresos altos (33-35). Asimismo, en el dominio de caminar como medio de transporte, en contraste con otros países donde se observa una disminución en las prevalencias (33), en Colombia la actividad física en este dominio ha aumentado. El dominio de montar en bicicleta no muestra un cambio de patrón en 
este período de cinco años y es importante resaltar que una de cada 10 personas reportó montar en bicicleta, al menos, 10 minutos seguidos.

En Colombia, este aumento en las prevalencias de caminar como medio de transporte y la tendencia estable de montar en bicicleta como medio de transporte, pueden estar parcialmente influenciados por factores como los cambios en los sistemas de transporte, los precios del combustible y las transformaciones en el medio ambiente físico construido. Los incrementos en el precio de la gasolina en el país, que aumentó de USD $\$ 0,72$ por litro en el 2004 a USD \$ 1,41 por litro en el 2010 (36), así como el de los precios del transporte público en los últimos 10 años, pueden ser factores que desincentivan el uso del transporte motorizado para recorridos cortos. Comparado con el de otros países de la región como México, Estados Unidos y Canadá, el precio del combustible en Colombia es uno de los más altos (36). Asimismo, la expansión de los sistemas de buses rápidos en las principales ciudades del país entre el 2000 y el 2010 (37) puede estar influenciando el cambio observado en el transporte activo. El diseño de este sistema de buses rápidos tiene el potencial de promover el transporte activo al tener paraderos fijos y requerir el uso de puentes peatonales para acceder a las estaciones de buses, y, asimismo, su construcción ha conducido a transformaciones en el medio ambiente físico que facilitan el uso del espacio público para caminar (38).

Por otra parte, aunque la tasa de motorización tiene una tendencia ascendente en Colombia en comparación con otras ciudades de Latinoamérica y el mundo, este crecimiento es bajo, lo que, sumado a las restricciones en la circulación de vehículos particulares, generalizadas en las principales ciudades del país, puede estar desincentivando el uso del vehículo particular y promoviendo caminar y usar la bicicleta como medios de transporte (3941). Además, se ha reportado una percepción de baja satisfacción con los sistemas de transporte público colectivo o masivo en todo el país, lo cual también podría incentivar el uso del transporte activo para trayectos cortos $(8,42)$.

Es importante contextualizar los patrones de actividad física y las desigualdades encontradas en este estudio en el marco de los programas y políticas nacionales para su promoción. En su Plan Nacional de Desarrollo, 2010-2014, el Gobierno Nacional estableció la promoción de la actividad física como un componente estratégico en la línea de "Igualdad de oportunidades para la prosperidad social" (14). En este sentido, se han implementado acciones dirigidas a la promoción de estilos de vida saludable, especialmente en grupos de población vulnerable, como el Programa de Hábitos y Estilos de Vida Saludable y la conformación de la Red Colombiana de Vías Activas y Saludables (Recovías). Dicha red fomenta la generación y el fortalecimiento de los programas de ciclovías recreativas, las cuales promueven la equidad y la inclusión social en la actividad física, y constituyen una oportunidad para incrementar sus niveles en el dominio de tiempo libre en poblaciones vulnerables (43). Asimismo, existen políticas del sector de transporte como el "pico y placa" (restricción a la circulación de vehículos motorizados según el número de la placa, la cual varía de acuerdo con la ciudad o municipio), que tienen como objetivo mitigar la congestión vehicular y, a su vez, promueven un aumento en la actividad física en el dominio de transporte.

Entre las principales limitaciones de este estudio está el diseño de corte transversal, el cual no permite establecer relaciones causales. En el análisis de tendencias en los cinco años, la medición se hizo únicamente en dos puntos en el tiempo, por lo que es de vital importancia observar estas tendencias en encuestas futuras. El uso del cuestionario IPAQ para la medición de la actividad física podría conducir a sobreestimar los niveles de dicha actividad; no obstante, en este estudio solo se incluyeron los dominios para los cuales se ha validado su uso en América Latina. Asimismo, el IPAQ únicamente toma en cuenta períodos de actividad física de 10 minutos continuos, por lo que los períodos menores y los correspondientes a trayectos cortos de transporte activo, no se toman en cuenta en este cuestionario. La población rural se excluyó, pero tiende a tener un menor nivel socioeconómico, lo cual puede haber llevado a subestimar las desigualdades analizadas. Por otra parte, la ausencia de datos sobre los ingresos de los hogares para el análisis de desigualdades por nivel socioeconómico, también constituye una limitante del estudio. Sin embargo, se contó con los datos de una encuesta con una muestra representativa que, por medio de una variable sustituta del nivel del Sisbén, permitió analizar estas desigualdades.

A pesar de estas limitaciones, el análisis evidenció diferencias preocupantes en el cumplimiento de las recomendaciones sobre actividad física claramente asociadas a los grupos sociales de mayor vulnerabilidad. Por otro lado, fue alentador 
constatar el aumento de la actividad de caminar como medio de transporte. Es importante que los programas nacionales y las futuras intervenciones para incrementar los niveles de actividad física en Colombia consideren las desigualdades por sexo y condición socioeconómica, así como los factores asociados a ellas, para contribuir de esta manera a la disminución de las diferencias en la salud.

\section{Agradecimientos}

Agradecemos los valiosos comentarios de los dos evaluadores anónimos, cuya contribución fue fundamental para mejorar el artículo.

\section{Financiación}

Para el análisis secundario tuvimos las siguientes fuentes de financiación: Silvia González obtuvo una beca otorgada por los Centers for Disease Control and Prevention, CDC. Andrea Ramírez fue financiada por el programa "Jóvenes Investigadores e Innovadores" del Departamento Administrativo de Ciencia, Tecnología e Innovación, Colciencias, convocatoria 525-2011. La ENSIN fue financiada por el Instituto Colombiano de Bienestar Familiar, el Instituto Nacional de Salud, Profamilia y el Ministerio de la Protección Social de la República de Colombia. La ENDS fue financiada por Profamilia, el Ministerio de la Protección Social de la República de Colombia, el Instituto Colombiano de Bienestar Familiar y la Agencia de los Estados Unidos para el Desarrollo Internacional (USAID).

\section{Conflicto de intereses}

Los autores del presente manuscrito declaran que no tienen conflictos de intereses.

\section{Referencias}

1. World Health Organization. Global Status Report in Noncommunicable Diseases 2010. Geneva: WHO; 2011.

2. Hosseinpoor AR, Bergen N, Mendis S, Harper S, Verdes E, Kunst A, et al. Socioeconomic inequality in the prevalence of noncommunicable diseases in low - and middle-income countries: Results from the World Health Survey. BMC Public Health. 2012;12:474. http://dx.doi.org/10.1186/14712458-12-474

3. Webber L, Kilpi F, Marsh T, Rtveladze K, Brown M, McPherson K. High rates of obesity and non-communicable diseases predicted across Latin America. PLoS One. 2012;7:e39589. http://dx.doi.org/10.1371/journal.pone.00 39589

4. Edwards P, Tsouros A. Promoting physical activity and active living in urban environments. The role of local governments. Copenhagen: World Health Organization; 2006.

5. World Health Organization. Global Health Risks: Mortality and burden of disease attributable to selected major risks. Geneva: WHO; 2009.
6. Lee IM, Shiroma EJ, Lobelo F, Puska P, Blair SN, Katzmarzyk PT. Effect of physical inactivity on major noncommunicable diseases worldwide: An analysis of burden of disease and life expectancy. Lancet. 2012;380:219-29. http://dx.doi.org/10.1016/S0140-6736(12)61031-9

7. Hallal PC, Andersen LB, Bull FC, Guthold R, Haskell W, Ekelund U. Global physical activity levels: Surveillance progress, pitfalls, and prospects. Lancet. 2012;380:247-57. http://dx.doi.org/10.1016/S0140-6736(12)60646-1

8. Instituto Colombiano de Bienestar Familiar (ICBF). Encuesta Nacional de la Situación Nutricional en Colombia. Bogotá: Instituto Colombiano de Bienestar Familiar; 2010.

9. Dumith SC, Hallal PC, Reis RS, Kohl HW. Worldwide prevalence of physical inactivity and its association with human development index in 76 countries. Prev Med. 2011;53:24-8. http://dx.doi.org/10.1016/j.ypmed.2011.02.017

10. Seclen-Palacín JA, Jacoby ER. Sociodemographic and environmental factors associated with sports physical activity in the urban population of Perú. Rev Panam Salud Pública. 2003;14:255-64. http://dx.doi.org/10.1590/S102049892003000900006

11. Florindo AA, Hallal PC, Moura EC, Malta DC. Practice of physical activities and associated factors in adults, Brazil, 2006. Rev Saúde Pública. 2009;43(Suppl.2):65-73. http://dx. doi.org/10.1590/S0034-89102009000900009

12. Salinas J, Vio F. Promoting health and physical activity in Chile: A policy priority. Rev Panam Salud Pública. 2003;14:281-8. http://dx.doi.org/10.1590/S1020-498920030 00900015

13. Comisión Económica para América Latina y el Caribe. CEPALSTAT. Bases de datos y publicaciones estadísticas. Fecha de consulta: 2 de febrero de 2013. Disponible en: http://estadisticas.cepal.org/cepalstat/WEB_CEPALSTAT/ Portada.asp.

14. Departamento Nacional de Planeación. Plan Nacional de Desarrollo 2010-2014: Prosperidad para todos. Bogotá: Departamento Nacional de Planeación; 2011.

15. Dávila EP, Quintero MA, Orrego ML, Ford ES, Walke H, Arenas MM, et al. Prevalence and risk factors for metabolic syndrome in Medellin and surrounding municipalities, Colombia, 2008-2010. Prev Med. 2013;56:30-4. http:// dx.doi.org/10.1016/j.ypmed.2012.10.027

16. Gómez LF, Duperly J, Lucumí DI, Gámez R, Venegas AS. Nivel de actividad física global en la población adulta de Bogotá (Colombia). Prevalencia y factores asociados. Gac Sanit. 2005;19:206-13.

17. Gómez LF, Moreno J, Gómez OL, Carvajal R, Parra DC. Physical activity and health-related quality of life among adult women in Cali, Colombia: A cross-sectional study. Qual Life Res. 2013;22:2351-8. http://dx.doi.org/10.1007/ s11136-013-0378-9

18. Organización Mundial de la Salud. Objetivos de desarrollo del milenio. Fecha de consulta: 4 de febrero de 2013. Disponible en: http://www.who.int/topics/millennium_ development_goals/gender/es/index.html.

19. Braveman P. Health disparities and health equity: Concepts and measurement. Annu Rev Public Health. 2006;27:16794. http://dx.doi.org/10.1146/annurev.publhealth.27.021405. 102103 
20. Profamilia. Encuesta Nacional de Demografía y Salud, ENDS 2010. Bogotá: Profamilia; 2010.

21. Instituto Colombiano de Bienestar Familiar. Encuesta Nacional de la Situación Nutricional ENSIN 2005. Bogotá: ICBF; 2005.

22. Hallal PC, Gómez LF, Parra DC, Lobelo F, Mosquera $\mathrm{J}$, Florindo AA, et al. Lessons learned after 10 years of IPAQ use in Brazil and Colombia. J Phys Act Health. 2010;7(Suppl.2):S259-64.

23. Bauman A, Ainsworth BE, Bull F, Craig CL, Hagstromer $\mathbf{M}$, Sallis JF, et al. Progress and pitfalls in the use of the International Physical Activity Questionnaire (IPAQ) for adult physical activity surveillance. J Phys Act Health. 2009;6(Suppl.1):S5-8.

24. Craig CL, Marshall AL, Sjöström M, Bauman AE, Booth ML, Ainsworth BE, et al. International physical activity questionnaire: 12-country reliability and validity. Med Sci Sports Exerc. 2003;35:1381-95.

25. U.S. Department of Health and Human Services (HHS). Physical activity guidelines for Americans 2008. Washington D.C: U.S. Department of Health and Human Services; 2012.

26. World Health Organization. Global recommendations on physical activity for health. Geneva: WHO; 2010.

27. The IPAQ Group. Guidelines for the data processing and analysis of the "International Physical Activity Questionnaire", 2009. Fecha de consulta: 21 de abril de 2014. Disponible en: http://www.ipaq.ki.se/scoring.htm.

28. Departamento Nacional de Planeación. Actualización de los criterios para la determinación, identificación y selección de beneficiarios de programas sociales. Documento CONPES Social 117. Bogotá: Departamento Nacional de Planeación; 2012.

29. Deddens JA, Petersen MR. Approaches for estimating prevalence ratios. Occup Environ Med. 2008;65:481, 501-6. http://dx.doi.org/10.1136/oem.2007.034777

30. Rothman K, Greenland S. Introduction to stratified analysis. Modern Epidemiology. Second edition. Philadelphia: Lippincott-Raven; 1998. p. 253-79.

31. Rothman K, Greenland S. Meta-analysis. Modern Epidemiology. Second edition. Philadelphia: Lippincott-Raven; 1998. p. 643-73.

32. Del Duca GF, Nahas MV, García LM, Mota J, Hallal PC, Peres MA. Prevalence and sociodemographic correlates of all domains of physical activity in Brazilian adults. Prev Med. 2013;56:99-102. http://dx.doi.org/10.1016/j.ypmed. 2012.11.007
33. Brownson RC, Boehmer TK, Luke DA. Declining rates of physical activity in the United States: What are the contributors? Annu Rev Public Health. 2005;26:421-43. http:// dx.doi.org/10.1146/annurev.publhealth.26.021304.144437

34. Petersen CB, Thygesen LC, Helge JW, Gronbaek M, Tolstrup JS. Time trends in physical activity in leisure time in the Danish population from 1987 to 2005. Scand $J$ Public Health. 2010;38:121-8. http://dx.doi.org/10. $1177 / 1403494809357098$

35. Alonso-Blanco $C$, Palacios-Cena $D$, Hernández-Barrera V, Carrasco-Garrido $P$, Jiménez-García R, Fernándezde-Las-Penas $\mathbf{C}$. Trends in leisure time and work-related physical activity in the Spanish working population, 19872006. Gac Sanit. 2012;26:223-30. http://dx.doi.org/10.1016/j. gaceta.2011.07.027

36. The World Bank. Pump price for gasoline. Fecha de consulta: 1 de agosto de 2013. Disponible en: http:// data.worldbank.org/indicator/EP.PMP.SGAS.CD/ countries $/ 1 \mathrm{~W}$ ?display=default.

37. Ministerio de Transporte de la República de Colombia, Oficina Asesora de Planeación. Transporte en cifras. Versión 2012. Bogotá: Ministerio de Transporte de la República de Colombia; 2012.

38. Mosquera J, Reis RS, Frank L, Ramírez-Marrero F, Welle B, Arriaga E, et al. Transport and health: A look at three Latin American cities. Cad Saúde Pública. 2013;29:654-66. http://dx.doi.org/10.1590/S0102-311X2013000800004

39. Dhondt S, Kochan B, Beckx C, Lefebvre W, Pirdavani A, Degraeuwe B, et al. Integrated health impact assessment of travel behaviour: Model exploration and application to a fuel price increase. Environ Int. 2013;51:45-58. http://dx.doi. org/10.1016/j.envint.2012.10.005

40. Secretaría Distrital de Movilidad. Informe de indicadores encuesta de movilidad de Bogotá 2011. Bogotá: Secretaría Distrital de Movilidad; 2012.

41. Secretaría Distrital de Movilidad. Resultados de la encuesta de movilidad de Bogotá 2011. Bogotá: Secretaría Distrital de Movilidad; 2013.

42. IPSOS Public Affairs. Percepción ciudadana sobre la calidad de vida en 10 ciudades colombianas. La encuesta de percepción de la Red Cómo Vamos 2012. Bogotá: IPSOS Public Affairs; 2013.

43. Torres A, Sarmiento OL, Stauber C, Zarama R. The ciclovia and ciclorruta programs: Promising interventions to promote physical activity and social capital in Bogota, Colombia. Am J Public Health. 2013;103:e23-30. http://dx. doi.org/10.2105/AJPH.2012.301142 